ESAIM: PROCEEDINGS, October 2011, Vol. 32, p. 149-162

E. Cancès, N. Crouseilles, H. Guillard, B. Nkonga, and E. Sonnendrücker, Editors

\title{
A SECOND ORDER ANTI-DIFFUSIVE LAGRANGE-REMAP SCHEME FOR TWO-COMPONENT FLOWS
}

\author{
Marie Billaud Friess ${ }^{1}$, Benjamin Boutin ${ }^{2}$, Filipa Caetano $^{3}$, Gloria Faccanoni ${ }^{4}$, \\ Samuel KokH ${ }^{5}$, Frédéric Lagoutière ${ }^{3,6}$ and Laurent Navoret ${ }^{7}$
}

\begin{abstract}
We build a non-dissipative second order algorithm for the approximate resolution of the one-dimensional Euler system of compressible gas dynamics with two components. The considered model was proposed in [1]. The algorithm is based on [8] which deals with a non-dissipative first order resolution in Lagrange-remap formalism. In the present paper we describe, in the same framework, an algorithm that is second order accurate in time and space, and that preserves sharp interfaces. Numerical results reported at the end of the paper are very encouraging, showing the interest of the second order accuracy for genuinely non-linear waves.

Résumé. Nous construisons un algorithme d'ordre deux et non dissipatif pour la résolution approchée des équations d'Euler de la dynamique des gaz compressibles à deux constituants en dimension un. Le modèle que nous considérons est celui à cinq équations proposé et analysé dans [1]. L'algorithme est basé sur [8] qui proposait une résolution approchée à l'ordre un et non dissipative au moyen d'un splitting de type Lagrange-projection. Dans le présent article, nous décrivons, dans le même formalisme, un algorithme d'ordre deux en temps et en espace, qui préserve des interfaces "parfaites" entre les constituants. Les résultats numériques rapportés à la fin de l'article sont très encourageants ; ils montrent clairement les avantages d'un schéma d'ordre deux pour les ondes vraiment non linéaires.
\end{abstract}

\section{INTRODUCTION}

The present paper investigates the simulation, on Cartesian grids, of flows involving two compressible components separated by a (sharp) interface. The discretization of the interface is achieved by means of an interface capture technique. Within this framework, the interface is located by the discontinuity locus of an additional parameter usually called a "color function".

We propose in this work an adaptation to the second order of the numerical scheme introduced in [8] for approximating the solution of the two-component flow model of [1]. This solver is based on a Lagrange-remap

${ }^{1}$ Université de Bordeaux, CEA, CNRS, CELIA, 351 Cours de la Libération, 33405 Talence (France); e-mail: billaud@celia.u-bordeaux1.fr

2 IRMAR - Université de Rennes 1, Campus de Beaulieu, 35042 Rennes (France); e-mail: benjamin.boutin@univ-rennes1.fr

3 Université Paris-Sud 11, Département de Mathématiques, CNRS UMR 8628, Bâtiment 425, 91405 Orsay (France); email: filipa.caetano@math.u-psud.fr \& frederic.lagoutiere@math.u-psud.fr

${ }^{4}$ IMATH, Université du Sud Toulon-Var, Bâtiment U, 83957 La Garde (France); e-mail: faccanon@univ-tln.fr

${ }^{5}$ DEN/DANS/DM2S/SFME/LETR, CEA Saclay, 91191 Gif-sur-Yvette (France); e-mail: samuel.kokh@cea.fr

6 Équipe-Projet SIMPAF, Centre de Recherche INRIA Futurs, Parc Scientifique de la Haute Borne, 40 Avenue Halley B.P. 70478, F-59658 Villeneuve d'Ascq (France)

7 Laboratoire MAP5 (UMR CNRS 8145), Université Paris Descartes, 45 rue des Saints Pères, 75270 Paris (France); email: laurent.navoret@parisdescartes.fr

(C) EDP Sciences, SMAI 2011 

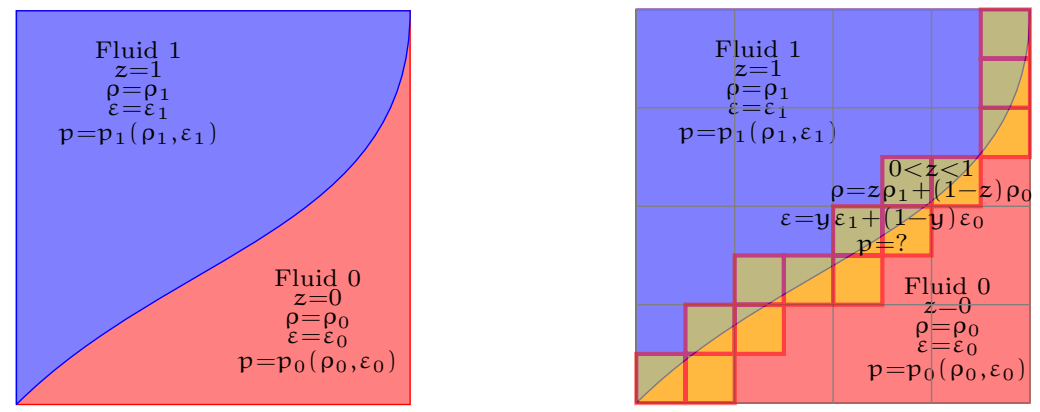

Figure 1: Two-fluid configuration with interface. Left: the two fluids are separated. Right: at the numerical level, there is a (numerical) mixing and a need to define a gas law for this mixture.

strategy. Second order schemes for compressible dynamics are extensively used (see [11]), even in the Lagrangeremap framework (see [6]). In the present contribution, the scheme is endowed with a special anti-diffusive treatment in the remap step which preserves the sharpness of the discretized interface. While the anti-diffusive mechanism allows to capture a very accurate profile for the contact discontinuity at the interface, it is not active for the resolution of genuinely non-linear waves. The purpose of this paper is to couple an anti-diffusive discretization technique for the color function with time and space second order schemes for the other unknowns, while the method of [8] only involves order one upwind methods. The resulting scheme remains conservative with respect to physically conserved parameters: global mass, partial masses, global momentum and energy.

The paper is structured as follows: we first briefly recall the five-equation system used for modeling the flow and the first order anti-diffusive Lagrange-remap method. Then, in section 4, we introduce the second order in space scheme, based on a second order Lagrange step and a second order remap step. Section 5 describes briefly the Runge-Kutta method used to achieve second order in time. Finally, we propose numerical results in section 6 .

\section{The FIVE-EQUATION MODEL}

In this section we briefly present, in dimension 1, the five-equation model derived and analyzed in [1], used for the description of interface flows composed of two compressible (immiscible) fluids. We adopt the same notations as in [8].

We introduce $\rho_{k}, p_{k}, \varepsilon_{k}$, the partial density, pressure and specific internal energy associated to each component of the flow $(k=0,1)$. We assume that each fluid is governed by an equation of state (EOS) of the form $\left(\rho_{k}, \varepsilon_{k}\right) \longmapsto p_{k}\left(\rho_{k}, \varepsilon_{k}\right)$. For each fluid we assume that there exists an entropy $s_{k}$ and we denote by $c_{k}=\sqrt{\left(\partial p_{k} / \partial \rho_{k}\right)_{s_{k}}}$ the sound speed of fluid $k$ (see $[1,8]$ for the modeling issues and details). The interface is localized thanks to the color function $(x, t) \longmapsto z(x, t)$ which is taken equal to 1 in the fluid 1 and 0 elsewhere. The global density $\rho$ and specific internal energy $\varepsilon$ of the two-component flow are related to the partial ones by

$$
\rho=z \rho_{1}+(1-z) \rho_{0} \quad \text { and } \quad \rho \varepsilon=z \rho_{1} \varepsilon_{1}+(1-z) \rho_{0} \varepsilon_{0} .
$$

In addition, we define the mass fraction $y_{k}$ by setting

$$
y=y_{1}=\frac{z \rho_{1}}{\rho} \quad \text { and } \quad y_{0}=1-y
$$


The five-equation model in one space dimension reads

$$
\left\{\begin{array}{l}
\partial_{t} \rho+\partial_{x}(\rho u)=0 \\
\partial_{t}(\rho y)+\partial_{x}(\rho y u)=0 \\
\partial_{t}(\rho u)+\partial_{x}\left(\rho u^{2}+p\right)=0 \\
\partial_{t}(\rho e)+\partial_{x}[u(\rho e+p)]=0 \\
\partial_{t} z+u \partial_{x} z=0 \\
p=p_{1}\left(\rho_{1}, \varepsilon_{1}\right)=p_{0}\left(\rho_{0}, \varepsilon_{0}\right)
\end{array}\right.
$$

where $e$ is the specific total energy given by $e=\varepsilon+u^{2} / 2$.

The first, third and fourth lines of System (1), express the conservation of total mass, total momentum and total energy. The fifth equation is the transport of the color function $z$ with the velocity $u$ common to both fluids (this model assumes that there is a unique velocity for the two fluids). In the sequel, we shall only consider initial conditions such that $z(x, t=0)=y(x, t=0) \in\{0,1\}$. Let us observe that for such initial values we have $z(x, t)=y(x, t) \in\{0,1\}$, for all $t>0$, therefore the second and the fifth lines become redundant. However, this relation will not be verified for the discrete approximate solution. Indeed, the numerical strategy proposed in this paper will generate regions in the computational domain where $0<z<1$ and $0<y<1$. Such regions are pure numerical artifacts that are a discretized representation of the sharp interface locus and cannot be considered as a meaningful physical mixture.

The Two-Perfect Gas Case. When both fluids are governed by perfect gas law $p_{k}=\left(\gamma_{k}-1\right) \rho_{k} \varepsilon_{k}$, with $\gamma_{\mathrm{k}}>1$, then the pressure $p$ verifies

$$
p=(\gamma-1) \rho \varepsilon, \quad \frac{1}{\gamma-1}=\frac{z}{\gamma_{1}-1}+\frac{1-z}{\gamma_{0}-1},
$$

and sound velocity $\mathrm{c}$ is defined by

$$
c^{2}=(\gamma-1) \sum_{k=0}^{1} \frac{y_{k}}{\gamma_{k}-1} c_{k}^{2}, \quad c_{k}^{2}=\frac{\gamma_{k}}{\rho_{k}} p .
$$

In the sequel we shall suppose that both fluids are perfect gas, however the present work is not restricted to this specific case and can be extended to more general equations of state using similar lines as in [1].

\section{LAGRANGE-REMAP ALGORITHM}

In this section, we review the first-order anti-diffusive Lagrange-remap scheme proposed in [8]. The Lagrangeremap scheme relies on a two-step splitting that decouples the acoustic effects from the material transport which are respectively associated with genuinely non-linear fields and linearly degenerate fields.

Let $X$ denote the Lagrangian coordinate, defined by

$$
(\partial X / \partial t)_{x}=u(X(x, t), t) \quad X(x, t=0)=x
$$

The first step is the Lagrangian step that consists in solving the Euler equations using Lagrangian coordinates. If we abuse notations and use the same variable names for both Lagrangian and Eulerian coordinates, this reads

$$
\rho \partial_{t}(1 / \rho)=\partial_{X} u, \quad \rho \partial_{t} u+\partial_{X} p=0, \quad \rho \partial_{t} e+\partial_{X}(p u)=0, \quad y_{t}=0, \quad z_{t}=0
$$

The second step remaps the variables back onto the original Eulerian frame. This step accounts for the material convection of the fluid.

This splitting strategy allows to provide a careful treatment with a special non-dissipative algorithm for the discretization of the transport effects. 
We refer to $[4,7]$ for a detailed review of the Lagrange-remap method and $[5,10]$ for an interpretation of the Lagrange-remap approach as a splitting of operators in the Eulerian frame.

Notation. Let $\Delta t>0$ and $\Delta x>0$ be a time step and a space step (they will be linked through a CourantFriedrichs-Lewy condition in the following). The space domain $\mathbb{R}$ is meshed with uniform cells of length $\Delta x$ indexed by $i \in \mathbb{Z}$.

For convenience, we keep the notations introduced in [9], avoiding the use of a time index $n$ : the numerical quantities in cell number $i$ are denoted in the following as $A_{i}$ at the beginning of the time step, $\widetilde{A}_{i}$ after the resolution of the Lagrange part, and $\widehat{A}_{i}$ after the remapping step.

For an interfacial flux variable $\left(\mathrm{F}_{i+1 / 2}\right)_{i \in \mathbb{Z}}$, let us call

$$
\llbracket \mathrm{F} \rrbracket_{i}=\mathrm{F}_{i+1 / 2}-\mathrm{F}_{i-1 / 2}
$$

the flux differences.

\subsection{Lagrange step $\mathfrak{L}^{1}$ (first order in space)}

We set $\tau=1 / \rho$ and use the following discretization for System (3)

$$
\begin{aligned}
& \rho_{i}\left(\widetilde{\tau}_{i}-\tau_{i}\right)=\lambda \llbracket u \rrbracket_{i}, \quad \rho_{i}\left(\widetilde{u}_{i}-u_{i}\right)=-\lambda \llbracket p \rrbracket_{i}, \quad \rho_{i}\left(\widetilde{e}_{i}-e_{i}\right)=-\lambda \llbracket p u \rrbracket_{i}, \\
& \widetilde{y}_{i}=y_{i}, \quad \widetilde{z}_{i}=z_{i},
\end{aligned}
$$

where $\lambda=\Delta t / \Delta x$.

Therefore, setting $\widetilde{\rho}_{i}=1 / \widetilde{\tau}_{i}, \widetilde{\rho u}_{i}=\widetilde{\rho}_{i} \widetilde{u}_{i}, \widetilde{\rho e}_{i}=\widetilde{\rho}_{i} \widetilde{e}_{i}, \widetilde{\rho y}_{i}=\widetilde{\rho}_{i} \widetilde{y}_{i}$ and defining the volume variation

$$
\mathrm{L}_{\mathfrak{i}}=1+\lambda \llbracket \mathfrak{u} \rrbracket_{i}
$$

we finally get the equivalent system in the Eulerian (conservative) variables

$$
\left\{\begin{array}{l}
\mathrm{L}_{i} \widetilde{\rho}_{i}=\rho_{i} \\
\mathrm{~L}_{i}(\widetilde{\rho u})_{i}=(\rho u)_{i}-\lambda \llbracket p \rrbracket_{i}, \\
\mathrm{~L}_{i}(\widetilde{\rho e})_{i}=(\rho e)_{i}-\lambda \llbracket p u \rrbracket_{i} \\
\widetilde{y}_{i}=y_{i} \\
\widetilde{z}_{i}=z_{i}
\end{array}\right.
$$

It remains to choose the numerical fluxes that appear in the right hand sides: $\llbracket \mathfrak{u} \rrbracket_{i}=u_{i+1 / 2}-u_{i-1 / 2}$, $\llbracket p \rrbracket_{i}=$ $p_{i+1 / 2}-p_{i-1 / 2}$ and $\llbracket p u \rrbracket_{i}=p_{i+1 / 2} u_{i+1 / 2}-p_{i-1 / 2} u_{i-1 / 2}$. They are defined following [3], where it is proved to lead to numerical discrete entropy inequalities:

$$
\begin{aligned}
& p_{i+1 / 2}=\frac{p_{i}+p_{i+1}}{2}-\frac{1}{2}(\rho c)_{i+1 / 2}\left(u_{i+1}-u_{i}\right), \\
& u_{i+1 / 2}=\frac{u_{i}+u_{i+1}}{2}-\frac{1}{2} \frac{1}{(\rho c)_{i+1 / 2}}\left(p_{i+1}-p_{i}\right),
\end{aligned}
$$

where

$$
(\rho c)_{i+1 / 2}=\sqrt{\max \left(\left(\rho c^{2}\right)_{i},\left(\rho c^{2}\right)_{i+1}\right) \min \left(\rho_{i}, \rho_{i+1}\right)} .
$$

Here $\left(\rho c^{2}\right)_{i}$ is computed thanks to (2). The Courant-Friedrichs-Lewy stability condition for this step is

$$
\frac{\Delta \mathrm{t}}{\Delta x} \max _{i \in \mathbb{Z}}\left((\rho c)_{i+1 / 2} / \min \left(\rho_{i}, \rho_{i+1}\right)\right) \leqslant 1 .
$$




\subsection{Remap step $\mathfrak{R}^{1}$ (first order in space)}

For the remap step, we use the general form (see $[5,7,8]$ )

$$
\left\{\begin{array}{l}
\widehat{\rho}_{i}=\widetilde{\rho}_{i}-\lambda \llbracket \widetilde{\rho} u \rrbracket_{i}+\lambda \widetilde{\rho}_{i} \llbracket u \rrbracket_{i}, \\
\widehat{\rho y}_{i}=\widetilde{\rho y}_{i}-\lambda \llbracket \widetilde{\rho y} u \rrbracket_{i}+\lambda \widetilde{\rho y}_{i} \llbracket u \rrbracket_{i}, \\
\widehat{\rho u}_{i}=\widetilde{\rho u}_{i}-\lambda \llbracket \widetilde{\rho u} u \rrbracket_{i}+\lambda \widetilde{\rho u}_{i} \llbracket u \rrbracket_{i}, \\
\widehat{\rho e}_{i}=\widetilde{\rho e}_{i}-\lambda \llbracket \widetilde{\rho e u} \rrbracket_{i}+\lambda \widetilde{\rho e}_{i} \llbracket u \rrbracket_{i}, \\
\widehat{z}_{i}=\widetilde{z}_{i}-\lambda \llbracket \widetilde{z} u \rrbracket_{i}+\lambda \widetilde{z}_{i} \llbracket u \rrbracket_{i} .
\end{array}\right.
$$

In this scheme, the flux differences $\llbracket u \rrbracket_{i}$ are those defined in the Lagrangian step. However, all the fluxes $\widetilde{\rho}_{i+1 / 2}, \widetilde{\rho u}_{i+1 / 2}, \widetilde{\rho e}_{i+1 / 2}, \widetilde{\rho y}_{i+1 / 2}$ and $\widetilde{z}_{i+1 / 2}$ remain to be defined. Following [5] and [8], we first take advantage of the anti-diffusive technology to compute the fluxes $\widetilde{z}_{i+1 / 2}$.

Note that the global Lagrange-remap scheme reads

$$
\left\{\begin{array}{l}
\widehat{\rho}_{i}=\rho_{i}-\lambda \llbracket \widetilde{\rho} u \rrbracket_{i}, \\
\widehat{\rho y}_{i}=\rho_{i} y_{i}-\lambda \llbracket \widetilde{\rho y} u \rrbracket_{i}, \\
\widehat{\rho u}_{i}=\rho_{i} u_{i}-\lambda \llbracket \widetilde{\rho u}+p \rrbracket_{i}, \\
\widehat{\rho e}_{i}=\rho_{i} e_{i}-\lambda \llbracket \widetilde{\rho e u}+p u \rrbracket_{i}, \\
\widehat{z}_{i}=z_{i}-\lambda \llbracket z u \rrbracket_{i}+\lambda z_{i} \llbracket u \rrbracket_{i},
\end{array}\right.
$$

and is thus conservative (except for the quantity $z$ ).

At the end of the time step, $\widehat{y}_{i}, \widehat{u}_{i}, \widehat{e}_{i}$ are computed as $\widehat{y}_{i}=\widehat{\rho y}_{i} / \widehat{\rho}_{i}, \widehat{u}_{i}=\widehat{\rho u}_{i} / \widehat{\rho}_{i}, \widehat{e}_{i}=\widehat{\rho e}_{i} / \widehat{\rho}_{i}$.

\subsubsection{Anti-diffusive flux for $z$}

The principle of the anti-diffusive scheme is to take the flux as downwinded as possible, but under some constraints guaranteeing stability and consistency: for instance, if $\boldsymbol{u}_{i+1 / 2}$ and $\boldsymbol{u}_{i-1 / 2}$ are positive, it means that $\widetilde{z}_{i+1 / 2}$ should be the nearest value to $\widetilde{z}_{\mathfrak{i}+1}=z_{i+1}$ that keeps the scheme consistent and stable. Let us precise the consistency and stability conditions.

Let us define $m_{i+1 / 2}^{z}=\min \left(z_{i}, z_{i+1}\right)$ and $M_{i+1 / 2}^{z}=\max \left(z_{i}, z_{i+1}\right), m_{i+1 / 2}^{y}=\min \left(y_{i}, y_{i+1}\right)$ and $M_{i+1 / 2}^{y}=$ $\max \left(y_{i}, y_{i+1}\right)$ and

$$
\begin{aligned}
d_{i+1 / 2} & =\frac{\left(\widetilde{\rho}_{0}\right)_{i+1 / 2} m_{i+1 / 2}^{y}}{\left(\widetilde{\rho}_{1}\right)_{i+1 / 2}\left(1-m_{i+1 / 2}^{y}\right)+\left(\widetilde{\rho}_{0}\right)_{i+1 / 2} m_{i+1 / 2}^{y}}, \\
D_{i+1 / 2} & =\frac{\left(\widetilde{\rho}_{0}\right)_{i+1 / 2} M_{i+1 / 2}^{y}}{\left(\widetilde{\rho}_{1}\right)_{i+1 / 2}\left(1-M_{i+1 / 2}^{y}\right)+\left(\widetilde{\rho}_{0}\right)_{i+1 / 2} M_{i+1 / 2}^{y}},
\end{aligned}
$$

where the fluxes $\left(\widetilde{\rho}_{0}\right)_{i+1 / 2}$ and $\left(\widetilde{\rho}_{1}\right)_{i+1 / 2}$ will be defined later (and as upwind fluxes). Now

- If $u_{i+1 / 2}>0$ and $u_{i-1 / 2}>0$, we set

$$
\begin{aligned}
& a_{i+1 / 2}=z_{i}+\left(z_{i}-M_{i-1 / 2}^{z}\right)\left(\frac{1}{\lambda u_{i+1 / 2}}-\frac{u_{i-1 / 2}}{u_{i+1 / 2}}\right), \\
& A_{i+1 / 2}=z_{i}+\left(z_{i}-m_{i-1 / 2}^{z}\right)\left(\frac{1}{\lambda u_{i+1 / 2}}-\frac{u_{i-1 / 2}}{u_{i+1 / 2}}\right),
\end{aligned}
$$


and

$$
\begin{aligned}
& b_{i+1 / 2}=z_{i}+\frac{\widetilde{\rho}_{i}\left(M_{i-1 / 2}^{y}-y_{i}\right)}{\left(\widetilde{\rho}_{1}\right)_{i}\left(1-M_{i-1 / 2}^{y}\right)+\left(\widetilde{\rho}_{0}\right)_{i} M_{i-1 / 2}^{y}}\left(\frac{u_{i-1 / 2}}{u_{i+1 / 2}}-\frac{1}{\lambda u_{i+1 / 2}}\right), \\
& B_{i+1 / 2}=z_{i}+\frac{\widetilde{\rho}_{i}\left(m_{i-1 / 2}^{y}-y_{i}\right)}{\left(\widetilde{\rho}_{1}\right)_{i}\left(1-m_{i-1 / 2}^{y}\right)+\left(\widetilde{\rho}_{0}\right)_{i} m_{i-1 / 2}^{y}}\left(\frac{u_{i-1 / 2}}{u_{i+1 / 2}}-\frac{1}{\lambda u_{i+1 / 2}}\right) .
\end{aligned}
$$

- If $u_{i+1 / 2}<0$ and $u_{i+3 / 2}<0$, we set

$$
\begin{aligned}
& a_{i+1 / 2}=z_{i+1}-\left(z_{i+1}-M_{i+3 / 2}^{z}\right)\left(\frac{1}{\lambda u_{i+1 / 2}}+\frac{u_{i+3 / 2}}{u_{i+1 / 2}}\right), \\
& A_{i+1 / 2}=z_{i+1}-\left(z_{i+1}-m_{i+3 / 2}^{z}\right)\left(\frac{1}{\lambda u_{i+1 / 2}}+\frac{u_{i+3 / 2}}{u_{i+1 / 2}}\right),
\end{aligned}
$$

and

$$
\begin{aligned}
& b_{i+1 / 2}=z_{i+1}+\frac{\widetilde{\rho}_{i+1}\left(M_{i+3 / 2}^{y}-y_{i+1}\right)}{\left(\widetilde{\rho}_{1}\right)_{i+1}\left(1-M_{i+3 / 2}^{y}\right)+\left(\widetilde{\rho}_{0}\right)_{i+1} M_{i+3 / 2}^{y}}\left(\frac{u_{i+3 / 2}}{u_{i+1 / 2}}+\frac{1}{\lambda u_{i+1 / 2}}\right), \\
& B_{i+1 / 2}=z_{i+1}+\frac{\widetilde{\rho}_{i+1}\left(m_{i+3 / 2}^{y}-y_{i+1}\right)}{\left(\widetilde{\rho}_{1}\right)_{i+1}\left(1-m_{i+3 / 2}^{y}\right)+\left(\widetilde{\rho}_{0}\right)_{i+1} m_{i+3 / 2}^{y}}\left(\frac{u_{i+3 / 2}}{u_{i+1 / 2}}+\frac{1}{\lambda u_{i+1 / 2}}\right) .
\end{aligned}
$$

Now let us define the sets $I_{i+1 / 2}$ :

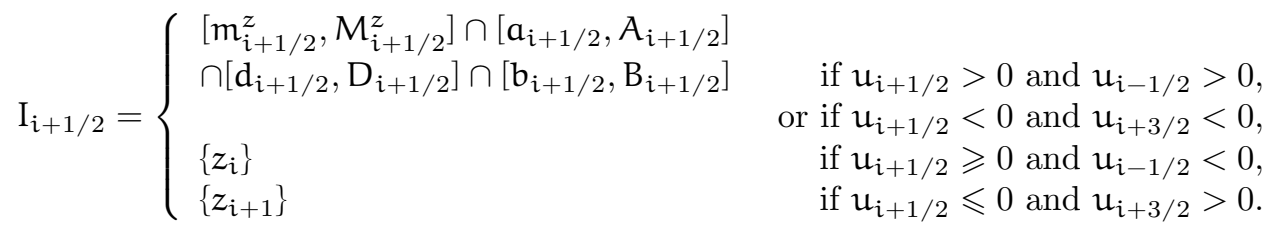

Then one has the following result (cf. [8]).

Proposition 3.1. Assume that all the fluxes of the remap step, as functions of the fluxes $\widetilde{z}_{\mathfrak{i}+1 / 2}$, are computed in accordance with formulae (10) and (12) in the following. Then, under the Courant-Friedrichs-Lewy condition $\left|u_{i+1 / 2}\right| \Delta t / \Delta x \leqslant 1$ for all $i \in \mathbb{Z}$, the sets $I_{i+1 / 2}$ are not empty and contain the upwind flux $z_{i}$ if $u_{i+1 / 2} \geqslant 0$, $z_{i+1}$ if $u_{i+1 / 2} \leqslant 0$. Furthermore, if $\widetilde{z}_{i+1 / 2} \in \mathrm{I}_{i+1 / 2}$ for every $i \in \mathbb{Z}$, the scheme is consistent and stable for $z$ and $\mathrm{y}$ in the sense that for all $i \in \mathbb{Z}$ one has

- $\widetilde{z}_{i+1 / 2} \in\left[m_{i+1 / 2}^{z}, M_{i+1 / 2}^{z}\right]$,

- $\widetilde{y}_{i+1 / 2} \in\left[m_{i+1 / 2}^{y}, M_{i+1 / 2}^{y}\right.$,

- if $u_{i+1 / 2} \geqslant 0$ then $\widehat{z}_{i} \in\left[m_{i-1 / 2}^{z}, M_{i-1 / 2}^{z}\right]$, and if $u_{i+1 / 2} \leqslant 0$ then $\widehat{z}_{i} \in\left[m_{i+1 / 2}^{z}, M_{i+1 / 2}^{z}\right]$,

- if $u_{i+1 / 2} \geqslant 0$ then $\widehat{y}_{i} \in\left[m_{i-1 / 2}^{y}, M_{i-1 / 2}^{y}\right]$, and if $u_{i+1 / 2} \leqslant 0$ then $\widehat{y}_{i} \in\left[m_{i+1 / 2}^{y}, M_{i+1 / 2}^{y}\right]$.

Choice of the flux $\widetilde{z}_{i+1 / 2}:$ the chosen flux $\widetilde{z}_{\mathfrak{i}+1 / 2}$ is the one that minimizes $\left|\widetilde{z}_{i+1 / 2}^{\text {do }}-\widetilde{z}_{i+1 / 2}\right|$ in the interval $I_{i+1 / 2}$, where $\widetilde{z}_{i+1 / 2}^{\text {do }}$ is the downwind value: $\widetilde{z}_{i+1 / 2}^{\text {do }}=z_{i+1}$ if $u_{i+1 / 2}>0, \widetilde{z}_{i+1 / 2}^{\text {do }}=z_{i}$ if $u_{i+1 / 2} \leqslant 0$. This is done in the following way. Let us denote $\mathrm{I}_{i+1 / 2}=\left[\omega_{i+1 / 2}, \Omega_{i+1 / 2}\right]$, the non-empty (under the Courant-FriedrichsLewy condition) consistency and stability interval for the flux $\widetilde{z}_{\mathfrak{i}+1 / 2}$. Then,

$$
\widetilde{z}_{i+1 / 2}=\left\{\begin{array}{l}
\omega_{i+1 / 2} \text { if } \widetilde{z}_{i+1 / 2}^{\mathrm{do}}<\omega_{i+1 / 2} \\
\widetilde{z}_{\mathfrak{i}+1 / 2}^{\mathrm{do}} \text { if } \widetilde{z}_{\mathfrak{i}+1 / 2}^{\mathrm{do}} \in\left[\omega_{i+1 / 2}, \Omega_{\mathfrak{i}+1 / 2}\right], \\
\Omega_{\mathfrak{i}+1 / 2} \text { if } \widetilde{z}_{\mathfrak{i}+1 / 2}^{\mathrm{do}}>\Omega_{\mathfrak{i}+1 / 2}
\end{array}\right.
$$


This flux will be denoted as $\widetilde{z}_{\mathfrak{i}+1 / 2}^{\text {ad }}$ in the following.

\subsubsection{Upwind flux for the other unknowns}

The fluxes for conservative unknowns are computed using the upwind choice for partial quantities $\left(\widetilde{\rho}_{k}\right)_{i+1 / 2}$, $\left(\widetilde{\rho_{k} \varepsilon_{k}}\right)_{i+1 / 2}, k=0,1$, and for $\widetilde{u}_{i+1 / 2}$ :

$$
\left(\left(\widetilde{\rho}_{k}\right)_{i+1 / 2}^{\text {up }},\left(\widetilde{\rho_{k} \varepsilon_{k}}\right)_{i+1 / 2}^{\text {up }}, \widetilde{u}_{i+1 / 2}^{\text {up }}\right)= \begin{cases}\left(\left(\widetilde{\rho}_{k}\right)_{i},\left(\widetilde{\rho_{k} \varepsilon_{k}}\right)_{i}, \widetilde{u}_{i}\right), & \text { if } u_{i+1 / 2}>0 \\ \left(\left(\widetilde{\rho}_{k}\right)_{i+1},\left(\widetilde{\rho_{k} \varepsilon_{k}}\right)_{i+1}, \widetilde{u}_{i+1}\right), & \text { if } u_{i+1 / 2} \leqslant 0\end{cases}
$$

We recall here that the partial densities are given through

$$
\left(\widetilde{\rho}_{1}\right)_{i}=\left\{\begin{array}{ll}
\frac{\widetilde{\rho y}_{i}}{z_{i}} & \text { if } z_{i} \neq 0, \\
0 & \text { otherwise }
\end{array} \quad \text { and } \quad\left(\widetilde{\rho}_{0}\right)_{i}= \begin{cases}\frac{\widetilde{\rho}_{i}\left(1-y_{i}\right)}{1-z_{i}} & \text { if } z_{i} \neq 1 \\
0 & \text { otherwise }\end{cases}\right.
$$

Finally the fluxes for conservative unknowns read

$$
\left\{\begin{array}{l}
\widetilde{\rho}_{i+1 / 2}=\widetilde{z}_{i+1 / 2}^{\text {ad }}\left(\widetilde{\rho}_{0}\right)_{i+1 / 2}^{\text {up }}+\left(1-\widetilde{z}_{i+1 / 2}^{\text {ad }}\right)\left(\widetilde{\rho}_{1}\right)_{i+1 / 2}^{\text {up }}, \\
(\widetilde{\rho y})_{i+1 / 2}=\widetilde{z}_{i+1 / 2}^{\text {ad }}\left(\widetilde{\rho}_{1}\right)_{i+1 / 2}^{\text {up }}, \\
(\widetilde{\rho \varepsilon})_{i+1 / 2}=\widetilde{z}_{i+1 / 2}^{\text {ad }}\left(\widetilde{\rho_{0} \varepsilon_{0}}\right)_{i+1 / 2}^{\text {up }}+\left(1-\widetilde{z}_{i+1 / 2}^{\text {ad }}\right)\left(\widetilde{\rho_{1} \varepsilon_{1}}\right)_{i+1 / 2}^{\text {up }}, \\
(\widetilde{\rho u})_{i+1 / 2}=\widetilde{\rho}_{i+1 / 2} \widetilde{u}_{i+1 / 2}^{\text {up }} \\
(\widetilde{\rho e})_{i+1 / 2}=(\widetilde{\rho \varepsilon})_{i+1 / 2}+1 / 2(\widetilde{\rho u})_{i+1 / 2} \widetilde{u}_{i+1 / 2}^{\text {up }} .
\end{array}\right.
$$

The Courant-Friedrichs-Lewy stability condition for this step is

$$
\frac{\Delta t}{\Delta x} \max _{i \in \mathbb{Z}}\left(\left|u_{i+1 / 2}\right|\right) \leqslant 1 .
$$

Thus the stability condition that we impose for the Lagrange-remap iterate is

$$
\frac{\Delta t}{\Delta x} \max _{i \in \mathbb{Z}}\left(\left|u_{i+1 / 2}\right|,(\rho c)_{i+1 / 2} / \min \left(\rho_{i}, \rho_{i+1}\right)\right) \leqslant 1 .
$$

We refer to $[8]$ for numerical results.

\section{SECOND ORDER IN SPACE}

The goal is now to develop a second order scheme in space, that will improve the numerical resolution of smooth solutions without breaking down the anti-diffusive properties.

To obtain second order accuracy in space, we use the classical MUSCL strategy (see [11] for example). For any variable $w$, the solution is approximated by a piecewise linear numerical solution (instead of the usual piecewise constant solution):

$$
\bar{w}_{i}(x)=w_{i}+\sigma_{i} x
$$

where $\sigma_{i}$ denotes a (numerical) slope of the solution (see Figure 2). To define these slopes, we use the slope limiter function:

$$
\sigma_{i}=\operatorname{minmod}\left(\frac{w_{i}-w_{i-1}}{\Delta x}, \frac{w_{i+1}-w_{i}}{\Delta x}\right),
$$




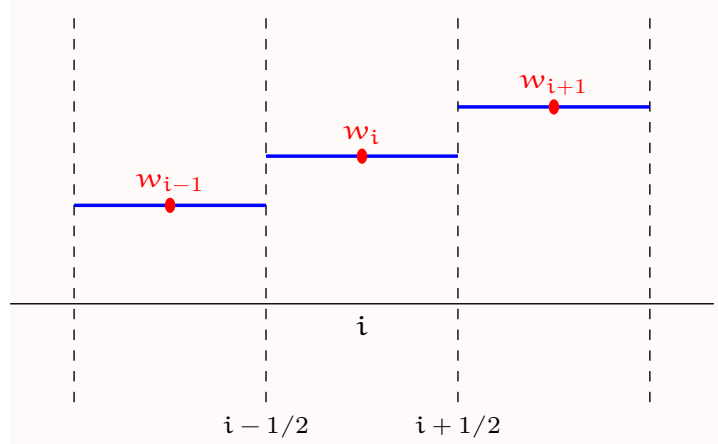

first order

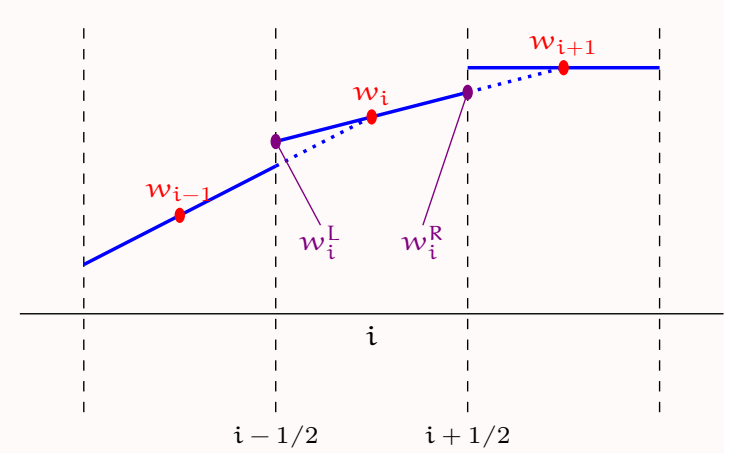

second order

Figure 2: MUSCL reconstruction (minmod).

where the minmod function ${ }^{1}$ is defined as:

$$
\operatorname{minmod}(a, b)= \begin{cases}a & \text { if }|a| \leqslant|b| \text { and } a b \geqslant 0, \\ b & \text { if }|b| \leqslant|a| \text { and } a b \geqslant 0, \\ 0 & \text { if } a b \leqslant 0,\end{cases}
$$

and is aimed at preventing from oscillations. This reconstruction enables to define a left value $w_{i}^{\mathrm{L}}=\bar{w}_{i}(-\Delta x / 2)$ and a right value $w_{i}^{R}=\bar{w}_{i}(\Delta x / 2)$, see Figure 2 .

In the next two subsections, we describe how it is incorporated into the Lagrange-remap scheme. The basic principle is to write any of the preceding first order fluxes $F_{i+1 / 2}$ as a function of the left and right values of the unknowns $w_{i}$ and $w_{i+1}: F_{i+1 / 2}=F\left(w_{i}, w_{i+1}\right)$, and to replace it here with $\mathrm{F}\left(w_{i}^{\mathrm{R}}, w_{i+1}^{\mathrm{L}}\right)$.

\subsection{Lagrange $\mathfrak{L}^{2}$ (second order in space)}

The second-order version of the Lagrange step (7)-(8) is written as follows:

$$
\begin{aligned}
& p_{i+1 / 2}=\frac{p_{i}^{R}+p_{i+1}^{L}}{2}-\frac{1}{2}(\rho c)_{i+1 / 2}\left(u_{i+1}^{L}-u_{i}^{R}\right), \\
& u_{i+1 / 2}=\frac{u_{i}^{R}+u_{i+1}^{L}}{2}-\frac{1}{2} \frac{1}{(\rho c)_{i+1 / 2}}\left(p_{i+1}^{L}-p_{i}^{R}\right), \\
& (\rho c)_{i+1 / 2}=\sqrt{\max \left(\left(\rho c^{2}\right)_{i}^{R},\left(\rho c^{2}\right)_{i+1}^{L}\right) \min \left(\rho_{i}^{R}, \rho_{i+1}^{L}\right),}
\end{aligned}
$$

where the values of $\rho^{L, R}, u^{L, R}$ and $p^{L, R}$ are defined from the values of $\rho, u$ and $p$ thanks to the affine reconstruction defined above. The values of $\left(\rho c^{2}\right)^{\mathrm{L}}$ and $\left(\rho c^{2}\right)^{R}$ are obtained with Equation $(2)$ and the values of $p^{\mathrm{L}, R}$ and $z$.

\footnotetext{
${ }^{1}$ It can be implemented as $\operatorname{minmod}(\mathbf{a}, \mathbf{b})=\max (0, \min (\mathbf{a}, \mathbf{b}))+\min (0, \max (\mathbf{a}, \mathbf{b}))$.
} 
Remark 4.1. Note that reconstructions of the mass fraction $y$ and the color function $z$ are not needed in this Lagrange step because $\widetilde{z}_{i}=z_{i}$ and $\widetilde{y}_{i}=y_{i}$.

\subsection{Remap $\mathfrak{R}^{2}$ (second order in space)}

For the remap step, we also reconstruct the left and the right values of all the quantities appearing in the fluxes, except for $z$. Indeed, in order to keep the interfaces sharp, we conserve the anti-diffusive flux for $z$ : $\widetilde{z}_{i+1 / 2}=\widetilde{z}_{i+1 / 2}^{\text {ad }}$ (see the end of Subsection 3.2.1). The algorithm is here based on a second order reconstruction of the partial densities:

$$
\left(\left(\widetilde{\rho}_{k}\right)_{i+1 / 2}^{\text {up2 }},\left(\widetilde{\rho_{k} \varepsilon_{k}}\right)_{i+1 / 2}^{\text {up2 }}, \widetilde{u}_{i+1 / 2}^{\text {up2 }}\right)= \begin{cases}\left(\left(\widetilde{\rho}_{k}\right)_{i}^{R},\left(\widetilde{\rho_{k} \varepsilon_{k}}\right)_{i}^{R}, \widetilde{u}_{i}^{R}\right) & \text { if } u_{i+1 / 2}>0 \\ \left(\left(\widetilde{\rho}_{k}\right)_{i+1}^{L},\left(\widetilde{\rho_{k} \varepsilon_{k}}\right)_{i+1}^{L}, \widetilde{u}_{i+1}^{L}\right) & \text { if } u_{i+1 / 2} \leqslant 0 .\end{cases}
$$

Recall that the stability property of Proposition 3.1 is true only if the fluxes are upwinded following Equation (12). Thus, in the vicinity of the interface, these first order fluxes have to be kept. Nevertheless one can choose second order fluxes away from it, when $z(1-z)=0$. This is done within the following procedure:

- If $z_{i-1}=z_{i}=z_{i+1}=z_{i+2}=0$ or 1 then the fluxes are second order:

$$
\begin{aligned}
& \widetilde{\rho}_{i+1 / 2}=\widetilde{z}_{i+1 / 2}^{\text {ad }}\left(\widetilde{\rho}_{0}\right)_{i+1 / 2}^{\text {up2 }}+\left(1-\widetilde{z}_{i+1 / 2}^{\text {ad }}\right)\left(\widetilde{\rho}_{1}\right)_{i+1 / 2}^{\text {up2 }}, \\
& (\widetilde{\rho y})_{i+1 / 2}=\widetilde{z}_{i+1 / 2}^{\text {ad }}\left(\widetilde{\rho}_{1}\right)_{i+1 / 2}^{\text {up } 2} \text {, } \\
& (\widetilde{\rho \varepsilon})_{i+1 / 2}=\widetilde{z}_{i+1 / 2}^{\text {ad }}\left(\widetilde{\rho_{0} \varepsilon_{0}}\right)_{i+1 / 2}^{\text {up2 }}+\left(1-\widetilde{z}_{i+1 / 2}^{\text {ad }}\right)\left(\widetilde{\rho_{1} \varepsilon_{1}}\right)_{i+1 / 2}^{\text {up2 }} \text {, } \\
& (\widetilde{\rho u})_{i+1 / 2}=\widetilde{\rho}_{i+1 / 2} \widetilde{u}_{i+1 / 2}^{u p 2} \text {, } \\
& (\widetilde{\rho e})_{i+1 / 2}=(\widetilde{\rho \varepsilon})_{i+1 / 2}+\frac{1}{2}(\widetilde{\rho u})_{i+1 / 2} \widetilde{u}_{i+1 / 2}^{u p 2}
\end{aligned}
$$

(but note that in this case $\widetilde{z}_{i+1 / 2}^{\text {ad }}=0$ or 1 ).

- If not, the fluxes are first order and given by formula (12).

\section{SECOND ORDER IN TIME}

To obtain second order accuracy in time, several strategies are possible. We propose here to use the RungeKutta method (RK2) (we also tested the method mentioned in [2], with no notable differences on numerical results).

The Runge-Kutta (RK2 or Heun) method is classical but for the sake of completeness, we recall here its principle. It consists in writing System (9) under the (vector) form $\widehat{W}=W+\lambda G(W)$ and to replace $(4,5,9)$ here with

$$
\begin{aligned}
& \breve{W}=W+(\lambda / 2) G(W) \\
& \widehat{W}=W+\lambda G(\breve{W}) .
\end{aligned}
$$

It is clear that this scheme is still conservative (except for $z$ ).

Remark 5.1. To have both the interfaces sharp and the stability result of Proposition 3.1, we have to keep order 1 (non-dissipative) accuracy when $z$ is not equal to 0 nor to 1 . This is done by computing the first oder in time fluxes in the vicinity of the interface, in the manner described at the end of subsection 4.2.

\section{Numerical RESUlts}

We compare first order and second order schemes on the two-component Sod shock tube. This test is a Riemann problem with initial condition

$$
(\rho, u, p, z)(x, 0)=\left\{\begin{array}{l}
(1,0,1,1) \text { if } x \leqslant 1 / 2 \\
(1 / 8,0,1 / 10,0) \text { if } x>1 / 2
\end{array}\right.
$$


with $\gamma_{0}=2.4$ and $\gamma_{1}=1.4$. We observe the results at time $t=0.14$, so that the waves do not reach neither $x=0$ nor $x=1$, and the boundary conditions are easy to handle.

Figures 3, 4, 5 represent the density, pressure, velocity, color function and mass fraction profiles at the final time with 400 cells. Here, solutions obtained with the first order, second order in space and second order in both space and time antidissipative scheme are compared to the exact ones.

In the following, "iํ $\mathfrak{R}^{j} \mathfrak{T}^{\mathrm{k}}$ " ("LiRjTk" in the figures) means that the considered numerical solution has been computed with the (antidissipative if not precised) scheme composed of the Lagrange step of order $i$, remap step of order $j$ and time integration discretization of order $k$.

Figures 6 and 7 present zooms of these numerical results. We remark that the second order improves a lot the precision of the approximation, especially along the rarefaction wave and on the shock wave. Nevertheless, there is no notable difference on the contact discontinuity. Indeed, the contact discontinuity, which corresponds to the color function discontinuity, is treated using an anti-dissipative scheme. One should note the overshoot and undershoot at the contact discontinuity. This so-called "wall-heating" phenomenon is usually smoothed when using classical schemes (like upwind scheme). Here, since the scheme is anti-dissipative, this artefact is not diffused. However, these oscillations are generally bounded and for finer mesh this phenomenon vanishes and does not affect the solution convergence even for more complex EOS (see for example [8]). Furthermore, the Runge-Kutta scheme for the time integration seems to bring no additional precision, but this may be test-case dependent.

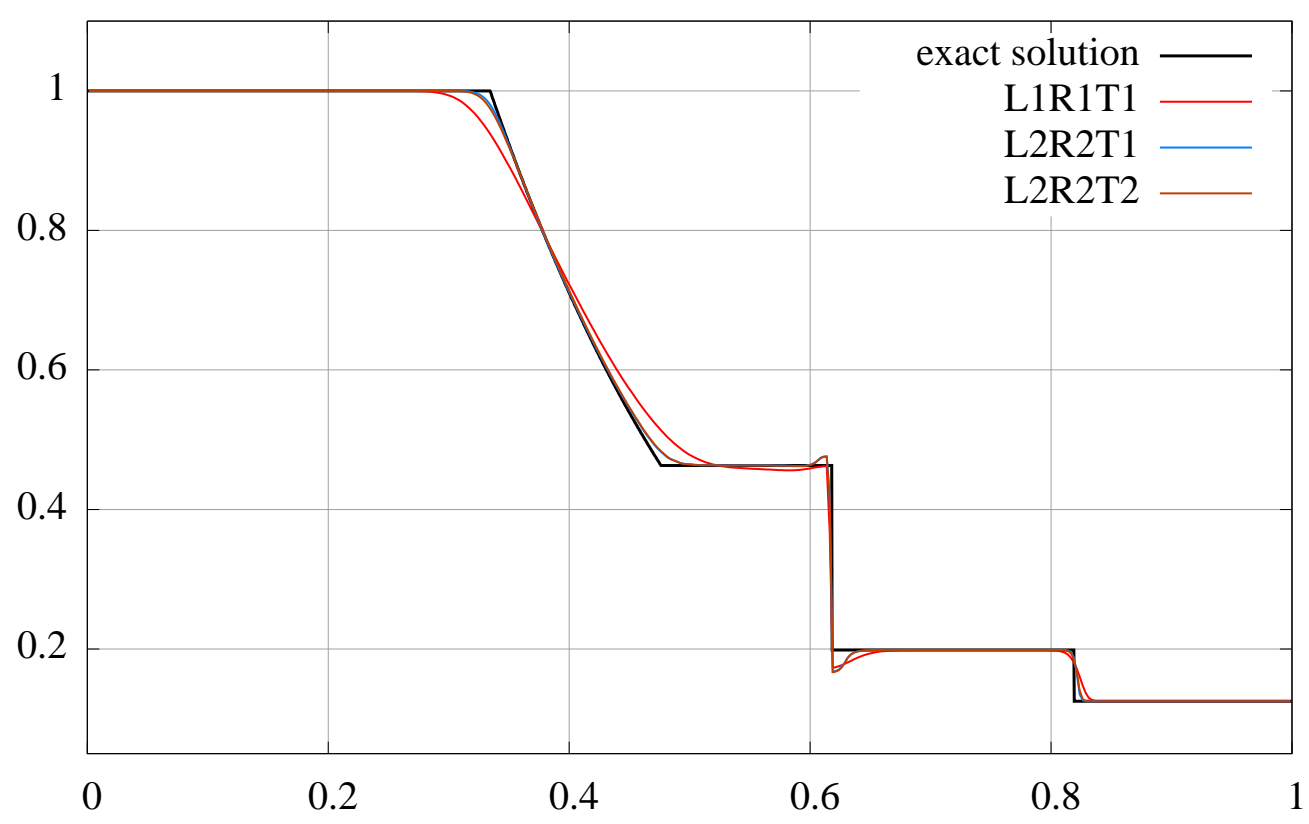

Figure 3: Two-component Sod shock tube: density.

In figure 8 , the $\mathrm{L}^{1}$ norm errors for the different quantities are depicted (in logarithmic scale) as a function of the space step for these three different schemes (with a ratio $\Delta t / \Delta x$ kept constant). In table 1 , we have reported the relative convergence orders (also compared to the classical first order and second order upwind schemes). Let us make some comments:

(1) For first and second order schemes, note that the anti-diffusive flux provides always better convergence rates than the upwind flux, for all the quantities. In particular for the mass fractions $y$ and $z$, the anti-diffusive flux enables to overcome order 1 errors (although these quantities are not smooth). 

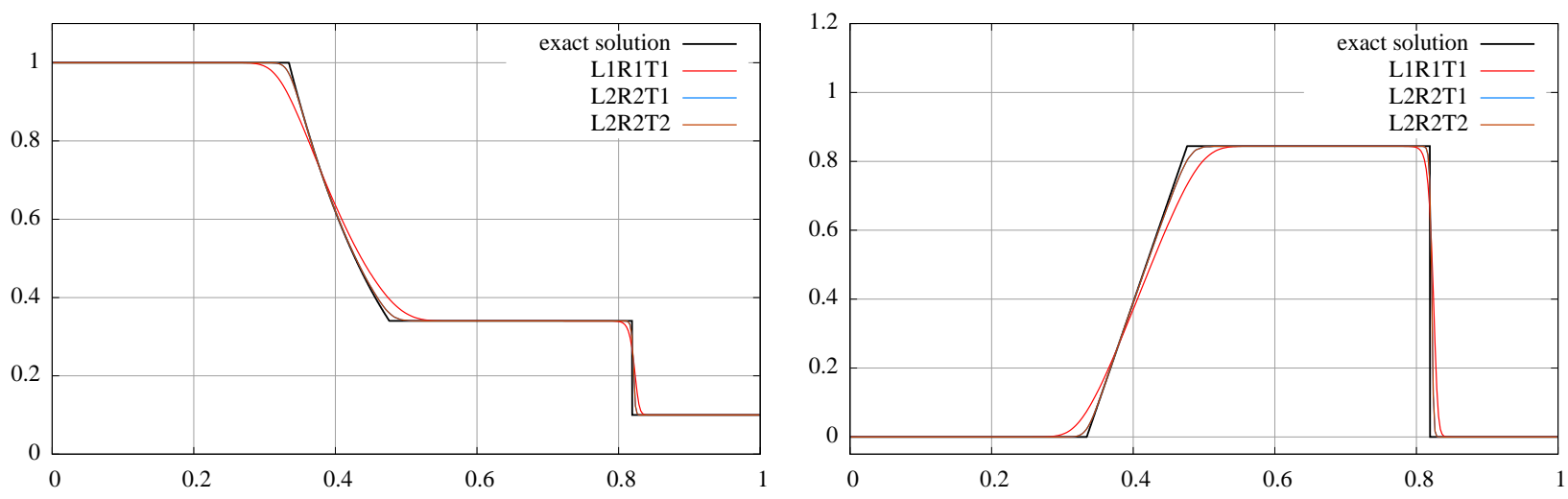

Figure 4: Two-component Sod shock tube: pressure (left) and velocity (right).
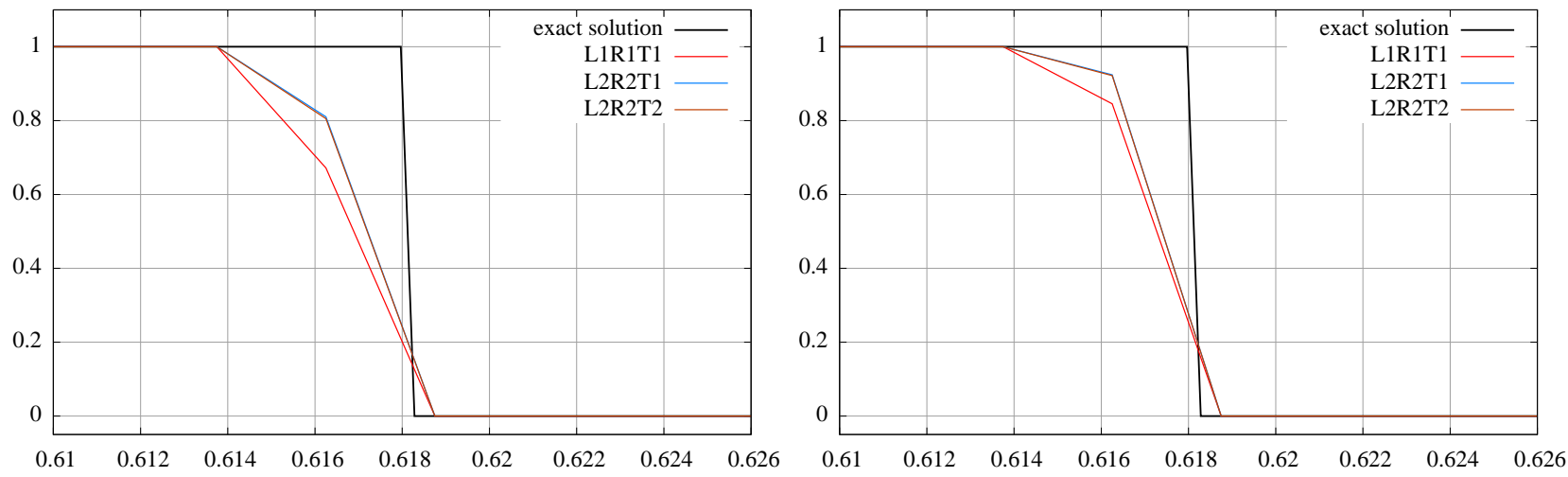

Figure 5: Two-component Sod shock tube: color function $z$ (left) and mass fraction $y$ (right).

(2) In comparison with the first order schemes, the second order methods improved the numerical convergence orders. However, the numerical orders that are reached are less or approximately equal to 1 instead of 2. This is partially due to the fact that the solution is discontinuous. We cannot expect to reach order 2 convergence. Furthermore, the order 1 convergence for the mass fractions is really the "best" that we can have. Besides, figure 8 shows that the error (as well as the the convergence rate) is also decreased when using second order schemes.

(3) We can also observe that the scheme $\mathfrak{L}^{2} \mathfrak{R}^{2} \mathfrak{T}^{1}$ gives better results than $\mathfrak{L}^{2} \mathfrak{R}^{1} \mathfrak{T}^{1}$ : more precisely, the second order method in the Lagrange step enables to improve the convergence rates of the mass fractions $y$ and $z$, while the second order in the remap step enables to improve the convergence rates of the density $\rho$, the velocity $z$ and the pressure $u$.

\section{Conclusion}

In this paper we explore a second order improvement of the antidissipative scheme for two-component compressible dynamics derived in [8]. Since this scheme is based on a Lagrange-projection strategy, the present 


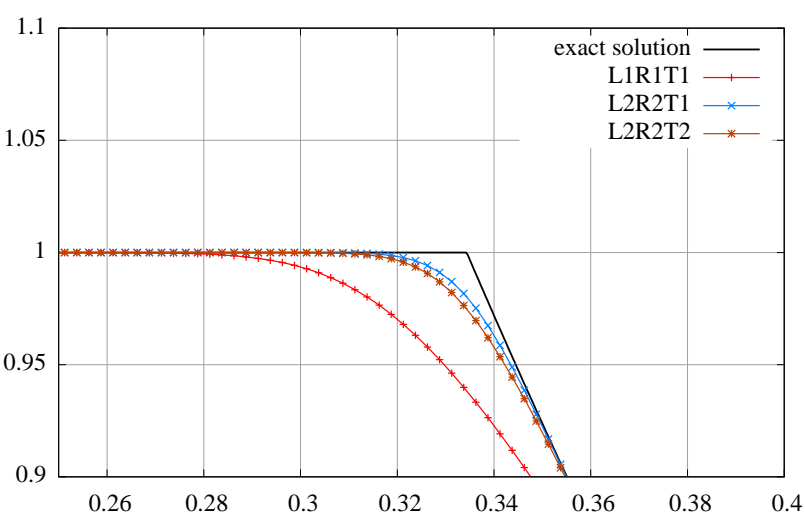

(a) "Head" of the rarefaction wave.

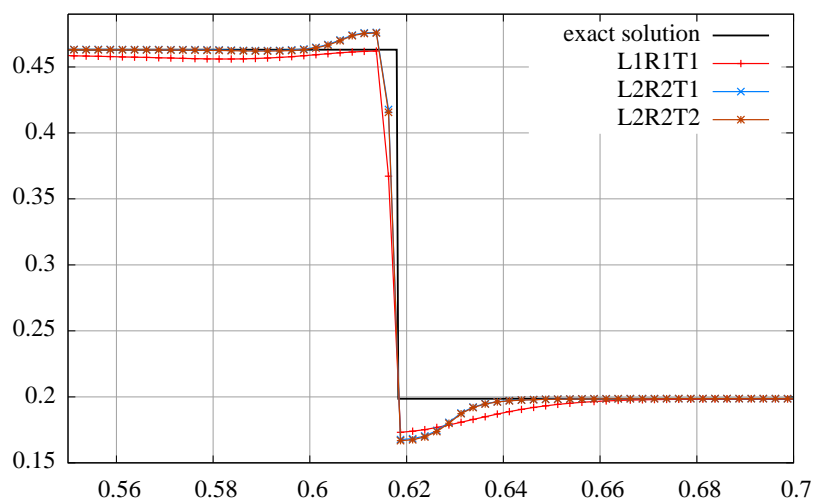

(b) Contact discontinuity.

Figure 6: Two-component Sod shock tube: density, zoom along the rarefaction wave (left) and on the contact discontinuity (right).

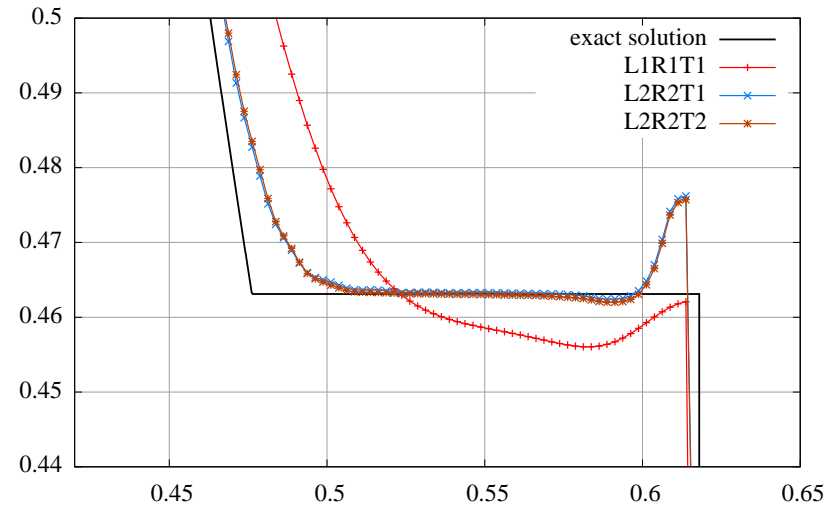

(a) Intermediate constant part.

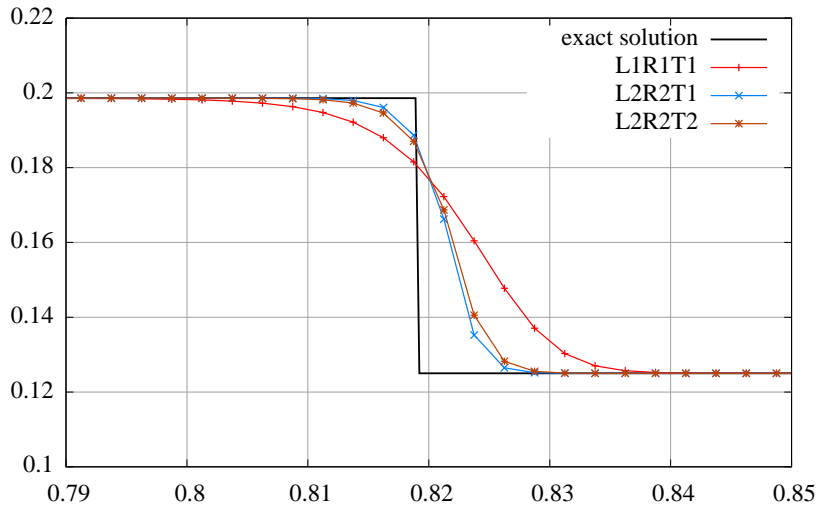

(b) Shock wave.

Figure 7: Two-component Sod shock tube: density, zoom between the rarefaction wave and the contact discontinuity (left) and on the shock wave (right).

paper proposes a second order Lagrange step as well as a second order remap step. This is done carefully, allowing to keep the former antidissipative properties for the color function and the mass fraction, which guaranties to have sharp interfaces between components. The preliminary numerical results reported in this paper show that the aim is reached: the computation of non-linear waves is improved while the interfaces remain sharp (located on only one cell).

Extension to the dimensions 2 and 3 are in progress, as well as the extension to the treatment of more than two components. 


\begin{tabular}{llccccc}
\hline & $z$ flux & $\rho$ & $u$ & $p$ & $y$ & $z$ \\
\hline $\mathfrak{L}^{1} \mathfrak{R}^{1} \mathfrak{T}^{1}$ & upwind & 0.63265 & 0.82033 & 0.77921 & 0.49348 & 0.51874 \\
$\mathfrak{L}^{1} \mathfrak{R}^{1} \mathfrak{T}^{1}$ & antidiffusive & 0.75714 & 0.82360 & 0.78031 & 1.12061 & 1.09585 \\
$\mathfrak{L}^{2} \mathfrak{R}^{2} \mathfrak{T}^{1}$ & upwind & 0.83484 & 1.03140 & 1.08738 & 0.69883 & 0.7288 \\
$\mathfrak{L}^{2} \mathfrak{R}^{2} \mathfrak{T}^{1}$ & antidiffusive & 1.08943 & 1.04647 & 1.10479 & 1.12100 & 1.25928 \\
\hline
\end{tabular}

Table 1: Two-component Sod shock tube - convergence orders

\section{REFERENCES}

[1] Grégoire Allaire, Sébastien Clerc, and Samuel Kokh. A five-equation model for the simulation of interfaces between compressible fluids. J. Comput. Phys., 181(2):577-616, 2002.

[2] Christophe Berthon. Stability of the MUSCL schemes for the Euler equations. Commun. Math. Sci., 3(2):133-157, 2005.

[3] Bruno Després. Inégalité entropique pour un solveur conservatif du système de la dynamique des gaz en coordonnées de Lagrange. C. R. Acad. Sci. Paris Sér. I Math., 324(11):1301-1306, 1997.

[4] Bruno Després. Lois de conservations eulériennes, lagrangiennes et méthodes numériques, volume 68 of Mathématiques 8 Applications (Berlin) [Mathematics \& Applications]. Springer-Verlag, Berlin, 2010.

[5] Bruno Després and Frédéric Lagoutière. Numerical resolution of a two-component compressible fluid model with interfaces. Prog. Comput. Fluid Dyn., 7(6):295-310, 2007.

[6] Frédéric Duboc, Cédric Enaux, Stéphane Jaouen, Hervé Jourdren, and Marc Wolff. High-order dimensionally split Lagrangeremap schemes for compressible hydrodynamics. C. R. Math. Acad. Sci. Paris, 348(1-2):105-110, 2010.

[7] Edwige Godlewski and Pierre-Arnaud Raviart. Numerical approximation of hyperbolic systems of conservation laws, volume 118 of Applied Mathematical Sciences. Springer-Verlag, New York, 1996.

[8] Samuel Kokh and Frédéric Lagoutière. An anti-diffusive numerical scheme for the simulation of interfaces between compressible fluids by means of a five-equation model. J. Comput. Phys., 229(8):2773-2809, 2010.

[9] Frédéric Lagoutière. Modélisation mathématique et résolution numérique de problèmes de fluides compressibles à plusieurs constituants. PhD thesis, Université Paris 6, 2000.

[10] Nicole Spillane. Méthode numérique pour les écoulements diphasiques de Baer-Nunziato. Master's thesis, Université Pierre et Marie Curie Paris 6, 2010.

[11] Eleuterio F. Toro. Riemann solvers and numerical methods for fluid dynamics. Springer-Verlag, Berlin, second edition, 1999. A practical introduction. 


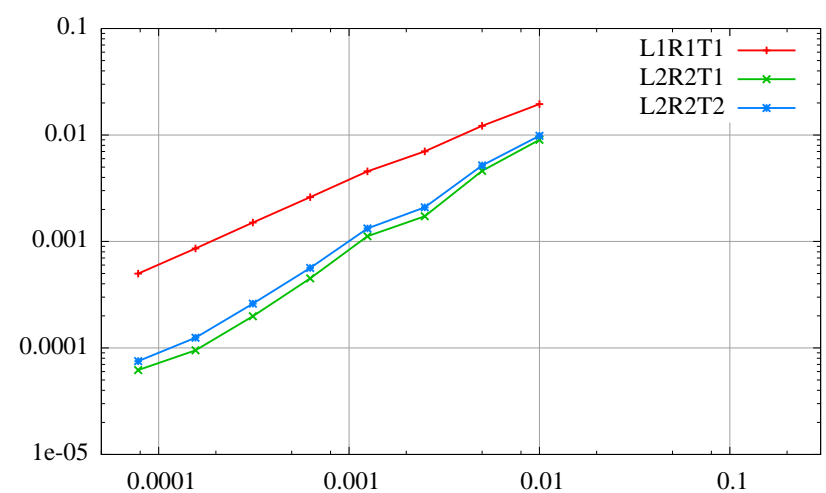

(a) Convergence in the $\mathrm{L}^{1}$ norm of the error for $\rho: \Delta x \mapsto \| \rho-$ $\rho_{\text {ex }} \|_{L^{1}}$ (logscale).

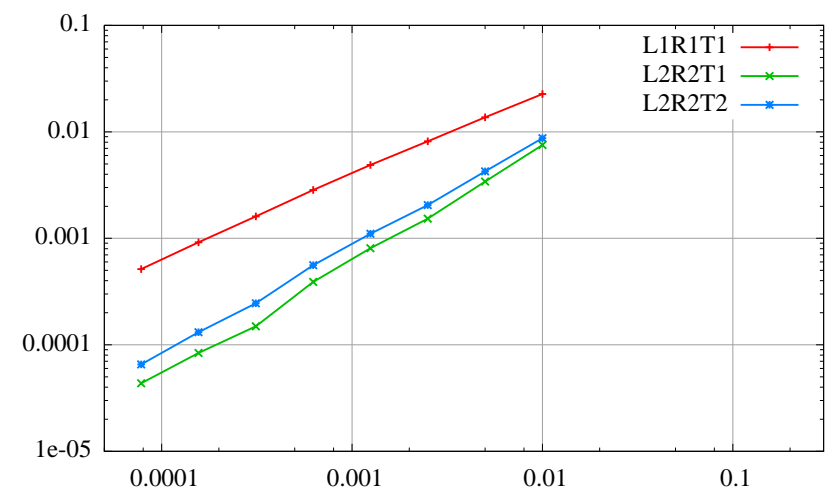

(c) Convergence in the $\mathrm{L}^{1}$ norm of the error for $\mathrm{p}: \Delta x \mapsto \| p-$ $p_{\text {ex }} \|_{L^{1}}$ (logscale).

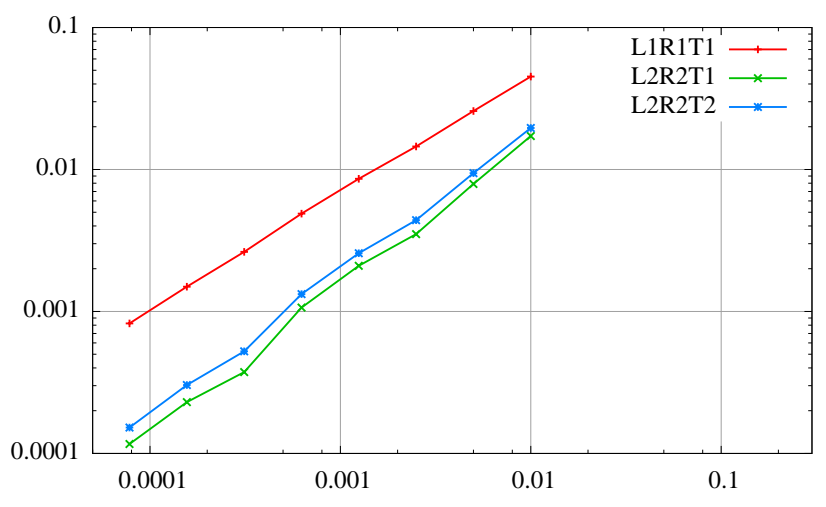

(b) Convergence in the $\mathrm{L}^{1}$ norm of the error for $\mathrm{u}: \Delta \mathrm{x} \mapsto \| \mathrm{u}-$ $u_{\text {ex }} \|_{L^{1}}$ (logscale).

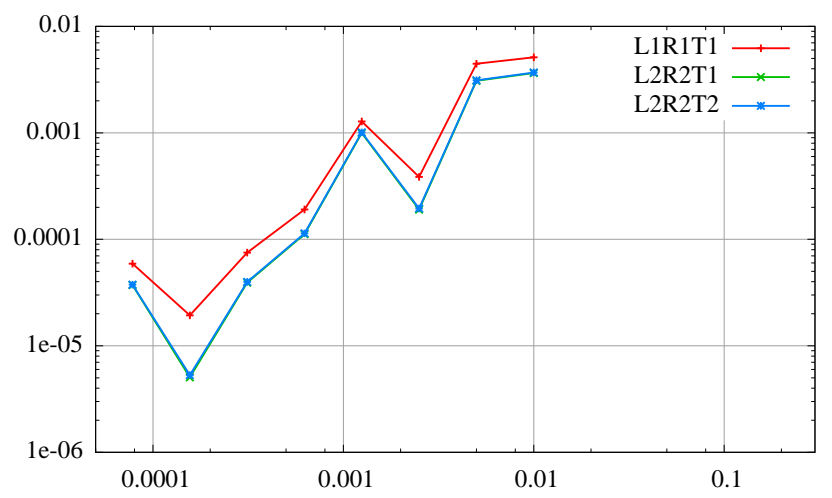

(d) Convergence in the $\mathrm{L}^{1}$ norm of the error for $\mathrm{y}: \Delta \mathrm{x} \mapsto \| \mathrm{y}-$ $y_{\text {ex }} \|_{L^{1}}$ (logscale).

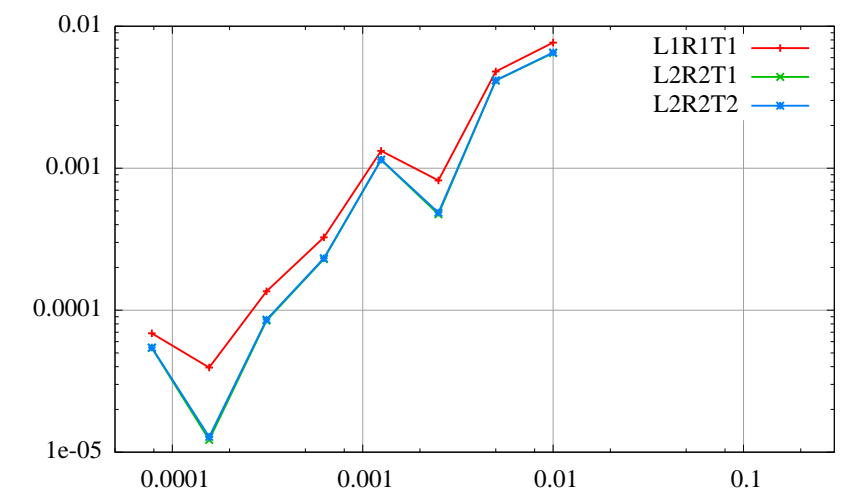

(e) Convergence in the $\mathrm{L}^{1}$ norm of the error for $z: \Delta x \mapsto \| z-$ $z_{\text {ex }} \|_{L^{1}}$ (logscale).

Figure 8: Two-component Sod tube: convergence curves for different quantities. 